\title{
X-band Accelerating Structure for Japan Linear Collider
}

\author{
T. Higo, M. Takao, K. Kubo and K. Takata \\ KEK, National Laboratory for High Energy Physics \\ Oho 1-1, Tsukuba-shi, Ibaraki-ken 305, Japan
}

\section{Abstract}

Candidates of the accelerating structure for realizing an operation in a multi-bunch mode in a linear collider were described. The external $Q$ value of the TM110- $\pi$ mode in a damped structure with slotted disk is very sensitive to the geometry. It was found impossible to reduce the $\mathrm{Q}$ value less than 100 for a structure with a beam hole radius of $a=3.75 \mathrm{~mm}$, while it can be as low as $\mathbf{1 2}$ for the structure with a small beam hole of $a=3 \mathrm{~mm}$. Furthermore, calculation for one of such a structure showed the reduction of the $Q$ value of the accelerating mode 20 percent and that of $R / Q 10$ percent. As a candidate of damped structure whose $Q$ value of the TM110 mode is insensitive to the geometry change, a so called crossed waveguide type was examined next. Only the TE111 type mode was found below $40 \mathrm{GHz}$ and no other mode was observed, indicating this structure a good candidate. An example of a so called detuned structure showed good damping within the following bunches.

\section{INTRODUCTION}

In order to obtain a high luminosity in a linear collider, the emittance of each bunch should be maintained small to make the spot size at interaction point small enough. The luminosity can still be increased if we can operate the linac in a multi-bunch mode. In the Japan linear collider (JLC), ten bunches, each contains $10^{10}$ particles and spaced $\mathrm{Tb}=1.4 \mathrm{~ns}$ apart with each other, are accelerated in an RF pulse using structures with $a / \lambda$ of $0.14[1]$. The energy of each bunch should reside in the acceptance of final focus in the presence of fundamental beam loading and also the long range longitudinal wake fields of higher modes. On the other hand, the transverse emittance of each bunch should also be preserved in the presence of long range dipole wake fields. In this paper, we discuss some possible cures against these multi-bunch effects in the linac focused on transverse case.

The simplest way of cure against the long range wake field is to damp the excited field in the structure before the arrival of the following bunch. This can be achieved in a so called damped structure. Two types of structures are discussed in the next section. Another type of cure is considered in the following section, where the excited wake field can be cancelled out by carefully tuning frequencies of all cells. This structure, so called detuned structure, is primitively described.

\section{DAMPED STRUCTURE}

Typical long range wake fields to be considered in the accelerating structure for JLC main linac are summarized in Table 1 together with the target values Q(Target) for each mode[2].
Table 1 Relevant long range wake fields.

\begin{tabular}{cccc}
\hline Mode & Freq. & Wake & Q(Target) \\
\hline Longitudinal & $(\mathrm{GHz})$ & $\left(\times 10^{15} \mathrm{~V} / \mathrm{C} / \mathrm{m}\right)$ & \\
TM010 & 11 & 0.5 & \\
TM020\&011 & 26 & 0.05 & \\
TM021 & 36 & 0.09 & 270 \\
\hline Transverse & $(\mathrm{GHz})$ & $\left(\times 10^{17} \mathrm{~V} / \mathrm{C}^{2} \mathrm{~m}^{2}\right)$ & \\
TM110 & 16 & 1.1 & 15 \\
TM111 & 26 & 0.18 & 38 \\
TM121 & 36 & 0.12 & 61 \\
\hline
\end{tabular}

\section{A. Slotted-disk type}

To achieve the target $Q$ value especially for TM1 10 mode, we investigated two-cell structure (DS-1) with a slotted disk at the middle of two cells[2]. Two waveguides are attached to the slots so that the TM110- $\pi$ mode can be damped out through these waveguides. In this configuration, resonant frequencies were calculated using the code MAFIA $[3]$ by varying the shorting position in the damping waveguides. The obtained tuning curves were analyzed by the authors $[2,4]$ and by $\mathrm{N}$. Kroll[5] and listed in Table 2. The results indicate that the structure which suffices the criterion of the $Q$ value is possible at least in this two-cell cavity.

Table 2. Analysis of damped structure DS-1.

\begin{tabular}{ccccc}
\hline & \multicolumn{2}{c}{ This author } & \multicolumn{2}{c}{ N. Kroll } \\
& $\mathrm{f}(\mathrm{GH})$ & $\mathrm{Q}$ & $\mathrm{f}(\mathrm{GHz})$ & $\mathrm{Q}$ \\
TM110 & 15 & $15-25$ & 15.4 & 11 \\
slot mode? & & & 16.1 & 7 \\
TM111 & 17 & $25-30$ & 17.0 & 15 \\
\hline
\end{tabular}

In order to damp the wake field in both polarizations, which is needed in actual linac structure, the other damping ports perpendicular to the previous ones should be equipped [6]. An example is shown schematically in Figure 1, where the direction of the damping ports at one disk is perpendicular to that at the next.

The obtained external $Q$ values of the TM110- $\pi$ mode for these structures are listed in the columns of DS- 2 and DS- 3 in Table 3. The parameters $\mathrm{W}, \mathrm{H} 1$ and $\mathrm{H} 2$ were varied to obtain as low $Q$ value as possible. As shown in the table, the $Q$ value is very sensitive to the structure, especially to the beam hole aperture. In the same table are listed the parameters of the two-cell cavity DS-1 of Table 1 and KEK-B scaled to $11.4 \mathrm{GHz}$ from the design of KEK-B factory [7]. These examples indicate a good damping for the structure with a small beam hole. The damping mechanism of this structure is not well understood but it seems that the low $Q$ value of TM1 10- $\pi$ is difficult to realize in the structure with a large beam hole. The beam hole 
radius of JLC accelerating structure is larger than $3.7 \mathrm{~mm}$ and it seems difficult to apply this type of structure.
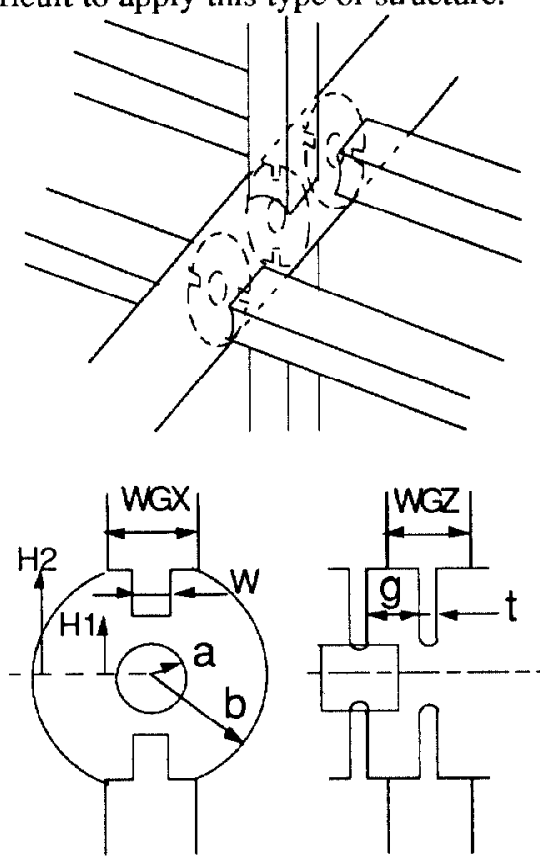

Fig. 1. Schematic drawing and geometry of damped structure DS-2 and DS-3.

Table 3. Parameters of various damped structures with slots in the disk.

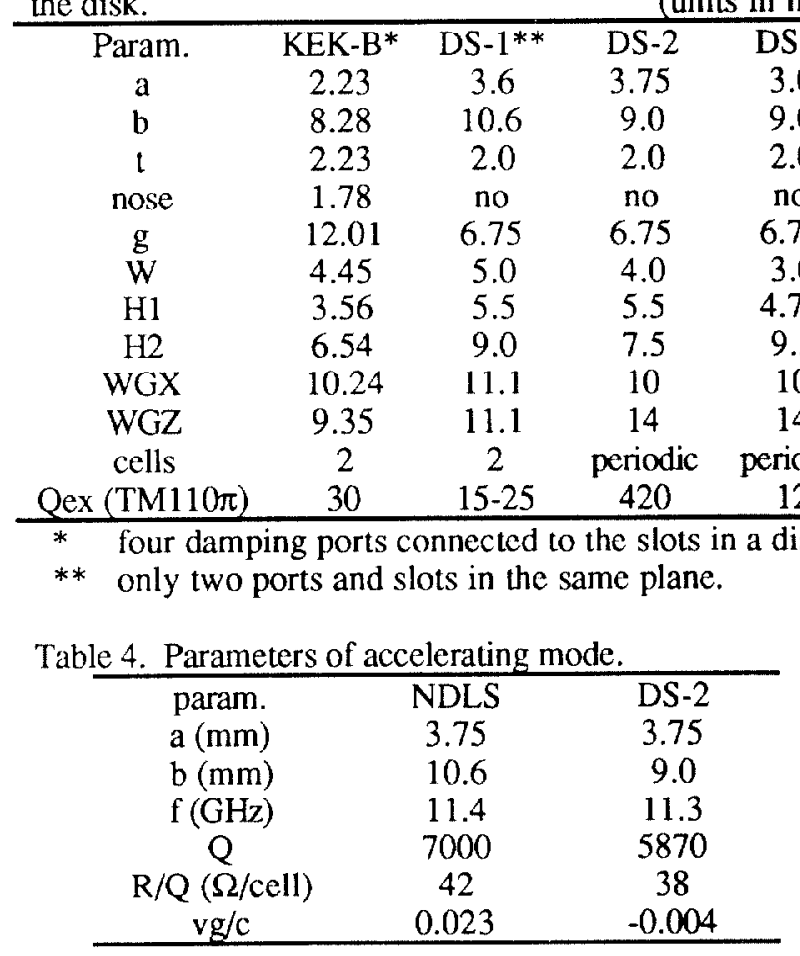

Parameters of the accelerating mode in $2 \pi / 3$ mode are also calculated by MAFIA for normal disk loaded structure (NDLS) and a damped structure (DS-2). The results are listed in Table 4[4]. The accelerating mode passband of DS-2 was found slightly backward because of a strong magnetic coupling through damping port and wide slot in the disk. This characteristic enables us to tune the group velocity of the accelerating modc without changing the beam hole aperturc. The reduction of the $Q$ value and the $R / Q$ are calculated to be about 10 and 20 percent, respectively. This pushes the peak power for the structure very high necessary to obtain a given accelerating gradient.

\section{B. Crossed waveguide type}

The damping of higher modes in the structure of crossed wavcguide type [8] is thought to be insensitive to the geometry change. In order to make all higher modes damped, a structure shown in Fig. 2 was examined. Each cell of the structure has 8 damping ports of $2 \mathrm{~mm} \times 11 \mathrm{~mm}$ in cross section extended to four directions and divided into two groups by a septum of its thickness $2 \mathrm{~mm}$ at the middle of the cell. The septum has a hole of radius $8 \mathrm{~mm}$ which determines a cell radius. The thickness of the disk is $2 \mathrm{~mm}$ and the beam hole radius is $3.5 \mathrm{~mm}$.

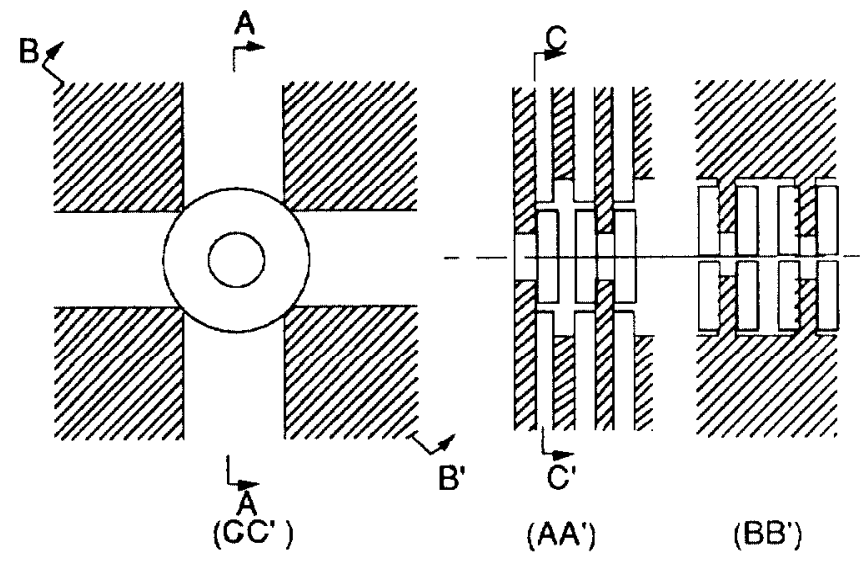

Fig. 2. Geometry of crossed waveguide type damped structure.

In Fig. 3 are shown the tuning curves obtained for a structure with these boundary conditions where such modes as TM110, TM111 and TM011-like ones can exist. The results were plotted as ( $\phi$,freq) diagram as suggested by $N$. Kroll[5]. According to Slater's formula[9], the parameters are related as

$$
\Phi=\frac{2}{\lambda \mathrm{g}} \mathrm{d} \cdot \mathrm{n}=\frac{1}{\pi} \operatorname{Arctan} \sum_{\mathrm{a}} \frac{\frac{1}{\mathrm{Qa}}}{\frac{\omega}{\omega \mathrm{a}}-\frac{\omega \mathrm{a}}{\omega}}
$$

where $\mathrm{d}$ is the position of the shorting plane, $\mathrm{n}$ the numbering of the branch, $Q_{a}$ and $\omega_{a}$ the $Q$ value and the angular frequency of a mode a. Here, the origin of the parameter $d$ is set at the center of the structure. In this calculation, the shorting plane of the waveguide were varied in only one direction (X direction). The parameter $\phi$ changes by $\pi$ if onc passes a resonance. All the steep changes of $\phi$ in the figures of TM110 and TM111 show the resonances composed of TE20 waveguide mode trapped in the waveguide of $\mathrm{Y}$ direction except the resonance at $25 \mathrm{GHz}$ in the figure of TM111. As the field pattern of this mode is similar to that of TE111 mode in the cell,we guess its $R / Q$ value small. In addition, the $Q$ value of this mode is as small as 36 obtained from the fitting of the tuning curve. From these consideration, the dangerous modes such as TM110 and TM111 are well damped in this type of damped structure. On the other hand, the steep resonances below $30 \mathrm{GHz}$ in the figure TM011 are attributed to 
the resonances in the waveguide of $\mathrm{Y}$ direction in TE10 waveguide mode. As the frequency of TM011 mode in normal disk loaded structure is about $26 \mathrm{GHz}[2]$ and the perturbation added from normal disk-loaded structure to this type of damped structure usually pushes the resonance frequency low, we conclude again that the TM011 mode is also well damped in this type of structure. As a final check, we should check the tuning curves by varying the shorting plane of all waveguides at once.
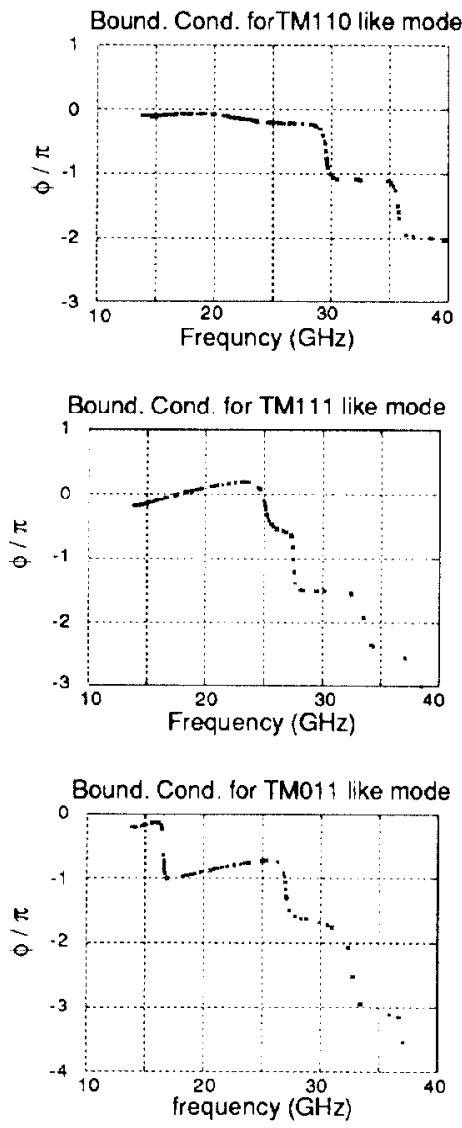

Fig. 3 Tuning curve of damped structure of crossed wageguide type.

\section{DETUNED STRUCTURE}

The wake field of TM110 mode should be reduced by two order of magnitude at the next bunch if we assume the same amount of damping criterion cited in the previous section. Simplest way to reduce the wake field at the following bunch is to tune a higher mode frequency to make a zero crossing at the following bunches, but it is difficult to realize this cancellation for more than a mode at once. Another extreme $[10]$ is to cancel out the wake field of each cell by varying the frequencies of the higher mode from cell to cell along a certain length of linac well shorter than the betatron wavelength.

To obtain a good damping at the next bunch and beyond that to the last bunch, a distribution similar to gaussian is desirable. An example of such a distribution is described as

$$
P(f)=\frac{T}{4}\left\{2 S(d f)+S\left(d f-\frac{1}{T}\right)+S\left(d f+\frac{1}{T}\right)\right\}
$$

for $\mid$ df $\mid<\frac{\delta f}{2}$, where df $\equiv f-f 0$ and $S(x) \equiv \frac{\operatorname{Sin}(\pi T x)}{\pi T x}$.

In Fig. 4 is shown the wake field excited by a bunch and integrated within 321 cells with frequencies distributed in the above formulus with $\mathrm{T}=2.4 \mathrm{~ns}$ and $\delta \mathrm{f} / \mathrm{f}=0.104$.

As the broad minimum at the next bunch (1.4ns) is mainly determined by $\delta \mathrm{f} / \mathrm{f}$, it can be controlled easily. The wake field after second bunch is well below $10^{-2}$.

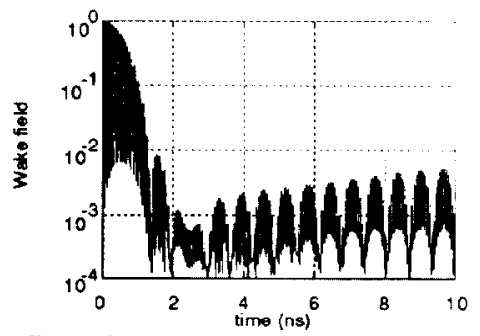

Fig. 4 Wake field integrated along 321 cells with $\delta \mathrm{f} / \mathrm{f}=0.104$ and $\mathrm{T}=2.4 \mathrm{~ns}$.

In the above estimation, we neglect those effects such as a finite coupling between cells, the successive excitation of wake field from cell to cell, different values of $R / Q$ among cells and the fabrication error of each cell frequency. Thesc effects should be carefully taken into account. Moreover, an actual geometry which provides us ten percent frequency distribution should be found. We should also estimate the effect of other higher modes.

\section{SUMMARY}

Some possible candidates of the structure for JLC which cures the effects due to the long range wake fields were examined. The damped structure with slots in the disk was found too sensitive to apply to the actual structure for JLC. A damped structure of crossed waveguide type was found good as for the characteristics of the higher modes. A detuned structure with its frequency distributed similar to a truncated gaussian shows a good cancellation of a higher mode.

\section{REFERENCES}

[1] K. Yokoya, Proc. First Workshop of Japan Linear Collider, 1989, Tsukuba.

[2] T. Higo et al., Proc. Linear Accelerator Conference, Alburquerque, 1990.

[3] K. Klatt et al., Proc. of the 1986 Linear Accelerator Conf., SLAC-Report-303,1986, p276-278.

[4] M. Takao, to be published.

[5] N. Kroll, private communication.

[6] T. Higo, Proc. Second Workshop of Japan Linear Collider, 1990, Tsukuba.

[7] M. Suetake et al., "Accelerator Design of KEK BFactory",KEK Report 90-24, 1991, p85.

[8] H. Deruyter et al., SLAC-PUB-5263, 1990.

[9] J. C. Slater, "Microwave Electronics", Van Nostrand, 1950

[10] H. Deruyter et al., SLAC-PUB-5322, 1990. 